\title{
Research on visible light indoor positioning algorithm based on fire safety
}

\author{
Mao Li, Feng Jiang ${ }^{*}$, Cong Pei \\ School of Computer and Information Engineering, \\ Central South University of Forestry and Technology, \\ Changsha 410004, China \\ *Corresponding author: jf09mail@126.com
}

\begin{abstract}
From the perspective of ensuring life safety, combined with the advantages of high-speed time response and energy conservation of white light emitting diodes (LEDs), the visible light indoor positioning algorithm based on fire safety is proposed in the paper. First, the model is designed which needs three LED lights arranged in a straight line and positioned in the geographically north direction on the top of the model. Then, the proposed algorithm is discussed and analyzed when the camera is located at the center of the model and facing north, when the camera is located at the center of the model and the angle is rotated, and when the camera is located at any position of the model, respectively. It can accurately calculate the current position of the camera, its response speed is fast and the positioning accuracy is high. Furthermore, this paper also verifies the practicability and reliability of the algorithm by designing the visible light indoor positioning system based on fire safety rescue in natural environment and smoke environment. The experimental results show that the positioning error does not exceed $0.70 \mathrm{~cm}$ in smoke environment.
\end{abstract}

Keywords: indoor positioning algorithm, camera, indoor positioning system.

\section{Introduction}

With the continuous development of the city, the buildings are getting higher and higher, and higher requirements are put forward for the fire safety protection technology. Once a fire breaks out in a high-rise building, the smog will cause the line of sight to decrease, which will reduce the efficiency of the rescue and cause great trouble to the rescue work. Therefore, it is particularly important to be able to quickly and accurately obtain the location of a trapped person, improve rescue efficiency, and reduce the risk of death. In addition, under normal circumstances, the indoor rescue environment of high-rise buildings is relatively harsh, and for search and rescue personnel, there are also great safety risks. In order to improve the safety factor of a trapped persons, it is always necessary to know the location of a trapped person which is a necessary function of modern fire safety equipment. 
In order to make fire rescue work in a timely, orderly and efficient manner, it is necessary to design an indoor positioning system [1] with high precision, high safety and low cost. At present, the indoor positioning systems are mainly studied from two aspects. The first aspect is the research of positioning technologies [2], and the second aspect is the research of positioning algorithms [ $\underline{3}]$. The mainstream direction of research is the study of location algorithms. After determining the use of a certain indoor positioning technology, it is necessary to introduce an effective positioning algorithm to achieve the expected indoor positioning accuracy. The existing indoor positioning algorithms include the LANDMARC algorithm using RFID technology [4]; the GIPS algorithm applied to university campuses and large indoor shopping malls [5]]; the passive infrared sensor tracking localization algorithm for smart home service systems [6]; and the indoor co-location algorithm for emergency situations [7] and car indoor positioning algorithm [ [ ] $]$, etc. These latest positioning algorithms have greater precision in their respective application scenarios. Similarly, the positioning accuracy has been significantly improved for three-dimensional spatial positioning. In the Ref. [9], the optimal receiver position is obtained by Tabu search, and the Ref. [10] proposes an improved chaotic particle swarm algorithm for indoor visible light localization system to improve positioning accuracy. Although the above indoor positioning algorithms improve the indoor positioning accuracy, the hardware cost and the complexity are both high.

From the perspective of ensuring life safety, combined with the high-speed time response and modulation characteristics of white LEDs, the visible light indoor positioning algorithm based on fire safety is proposed in the paper. Multiple LEDs are used as the transmitting end, and the camera is used as the receiving end [11]. The algorithm considers three situations when the camera has different positions and orientations in the indoor space. The image of the LED lights is captured by the camera to determine the current position of the camera, and the positioning accuracy is high. In addition, the reliability and practicability of the algorithm are also verified based on the visible light indoor positioning system. The experimental results show that the positioning error does not exceed $3 \mathrm{~cm}$.

\section{Visible light indoor positioning algorithm}

Based on the existing visible light indoor positioning algorithm, the positioning algorithm is proposed using white LED as the reference signal transmission source, and uses its modulation characteristics to realize communication [12]. The detection sensor uses the camera, and its accuracy requirements and speed requirements are what the algorithm needs to consider.

\subsection{Construction of optical model}

Considering the size of indoor space is $L \times W \times H$, the LED light sources are fixed to the top of the indoor space which are arranged in a straight line whose position points 


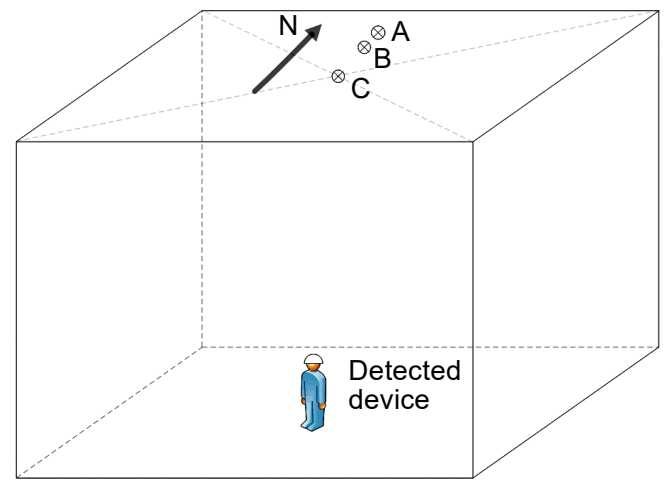

Fig. 1. LED positioning model.

to the geographical north direction, and the distance between adjacent two lights is not equal.

The position model is shown in Fig. 1. Assuming that the coordinates of the three LEDs are $A\left(x_{1}, y_{1}, H\right), B\left(x_{2}, y_{2}, H\right)$ and $C\left(x_{3}, y_{3}, H\right)$, respectively, the distance between $A$ and $B$ is $\Lambda_{1}$, and the distance between $B$ and $C$ is $\Lambda_{2}\left(\Lambda_{1}<\Lambda_{2}\right)$.

Assuming that the radius of the roof LED bulb is $r$, the maximum distance of the object to fully image the three LEDs is $\Lambda_{1}+\Lambda_{2}+2 r$, the height of the model is $H$, and the expression is as follows:

$$
f=\frac{(H-h) \varphi}{\Lambda_{1}+\Lambda_{2}+2 r}
$$

where $f$ is the focal length of the lens, $\varphi$ is the size of the lens model, and $h$ is the height of the camera from the ground.

When the lens needs to capture three LEDs completely anywhere in the indoor space, the lens is located in the corner of the indoor space considering the worst case. At this time, the lens needs to completely capture three LEDs, and the lens field of view (FOV) should be larger than $\mathrm{FOV}_{\text {min }}$ :

$$
\mathrm{FOV}_{\text {min }}=\sqrt{\left(\frac{L}{2}+\varepsilon_{1}\right)^{2}+\left(\frac{W}{2}+\varepsilon_{2}\right)^{2}}
$$

where $L$ is the length of the indoor space, $W$ is the width of the indoor space, $\varepsilon_{1}$ and $\varepsilon_{2}$ are the position error of LED installation. The actual field of view of the lens

$$
\mathrm{FOV}=\frac{H \varphi}{f}
$$

where $H$ is the height of the indoor space, $f$ is the focal length of the lens, and $\varphi$ is the size of the lens model. 


\subsection{Positioning algorithm}

When the camera is located in different positions of the indoor space, the camera can take the following three pictures, where $A^{\prime}, B^{\prime}$, and $C^{\prime}$ are the spots formed by the LED lights. Consider the following three situations.

\subsubsection{The camera is located in the center of the indoor space and faced north}

This position is specified as the initial origin of positioning, and the captured picture is shown in Fig. 2.

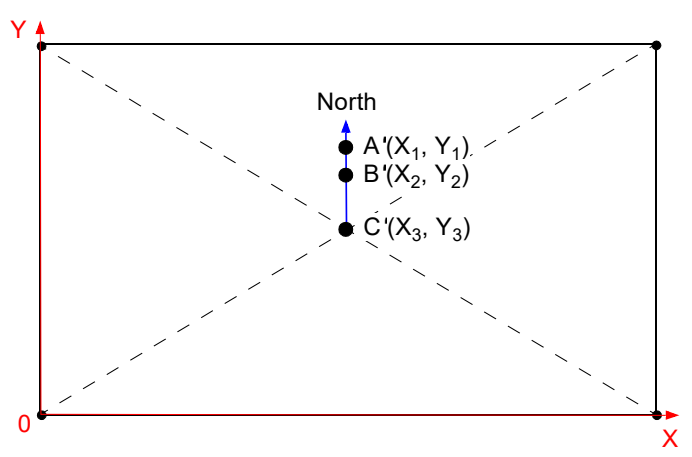

Fig. 2. The camera is located in the center of the indoor space and faced north.

Assuming that the distance between $A^{\prime}$ and $B^{\prime}$ is $\lambda_{1}$, and the distance between $B^{\prime}$ and $C^{\prime}$ is $\lambda_{2}$, then $\lambda_{1}<\lambda_{2}$. Set $A^{\prime}\left(X_{1}, Y_{1}\right), B^{\prime}\left(X_{2}, Y_{2}\right)$ and $C^{\prime}\left(X_{3}, Y_{3}\right)$. At this time, the relative deflection angle of the lens is $\theta$.

First calculate the distance between any two spots, and then determine which LED corresponds to each spot. Its distance $L_{1}, L_{2}$, and $L_{3}$ is expressed as follows:

$$
\begin{aligned}
& L_{1}=\sqrt{\left(X_{1}-X_{2}\right)^{2}+\left(Y_{1}-Y_{2}\right)^{2}} \\
& L_{2}=\sqrt{\left(X_{2}-X_{3}\right)^{2}+\left(Y_{2}-Y_{3}\right)^{2}} \\
& L_{3}=\sqrt{\left(X_{1}-X_{3}\right)^{2}+\left(Y_{1}-Y_{3}\right)^{2}}
\end{aligned}
$$

Further, since $\lambda_{1}<\lambda_{2}$, it can be judged that $A^{\prime}$ is a spot formed by the lamp $A, B^{\prime}$ is a spot formed by the lamp $B$, and $C^{\prime}$ is a spot formed by the lamp $C$.

As shown in Fig. 2, the spot $C^{\prime}$ of the lamp $C$ is at the center of the captured screen, which indicates that the camera is in the origin position at the time of detection, and the relative deviation angle of the detection camera (defined as the angle between the $\mathrm{Y}$-axis and the ray formed by the three LED spots in the captured picture) is 0 degrees, indicating that the camera is facing the geographical north direction. 


\subsubsection{The camera is located in the center of the room but the angle is rotated}

When the detection camera is located directly below the lamp $C$ and rotates around the $Z$ axis, the light source diagram is as shown in Fig. 3.

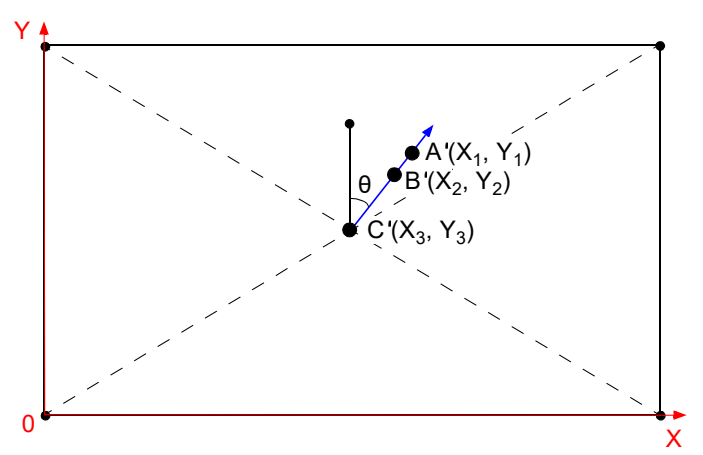

Fig. 3. The camera is located in the center of the room but the angle is rotated.

The relative deflection angle of the lens can be calculated from the two spot coordinates of $A^{\prime}$ and $C^{\prime}$ :

$$
\begin{aligned}
& \tan \theta=\frac{X_{1}-X_{3}}{Y_{1}-Y_{3}} \\
& \theta=\operatorname{atan} \frac{X_{1}-X_{3}}{Y_{1}-Y_{3}}
\end{aligned}
$$

The actual lens rotation direction is opposite, so the actual lens is rotated counterclockwise $\theta$, that is, a person faces the north-west angle $\theta$.

\subsubsection{The camera is located at the ground anywhere}

Analysis based on the above two cases, when it is in any position on the ground, the light source diagram is as shown in Fig. 4.

In order to calculate the position information of the camera at this time, it needs to be solved in three steps.

1) First calculate the current lens pointing (actual lens deflection angle). The relative deflection angle $\theta$ is calculated by Eq. (6), and the direction in which the actual lens rotates is relative, so the actual lens in Fig. 4 is rotated clockwise by $\theta$ (the actual lens deflection angle is $\theta$ ).

2) Then calculate the actual direction of motion and the motion step size of the lens. The direction in which the lens actually moves is the direction of the center point $M$ relative to the $C^{\prime}$ motion. Suppose the resolution of the camera is $320 \times 240$, so the 


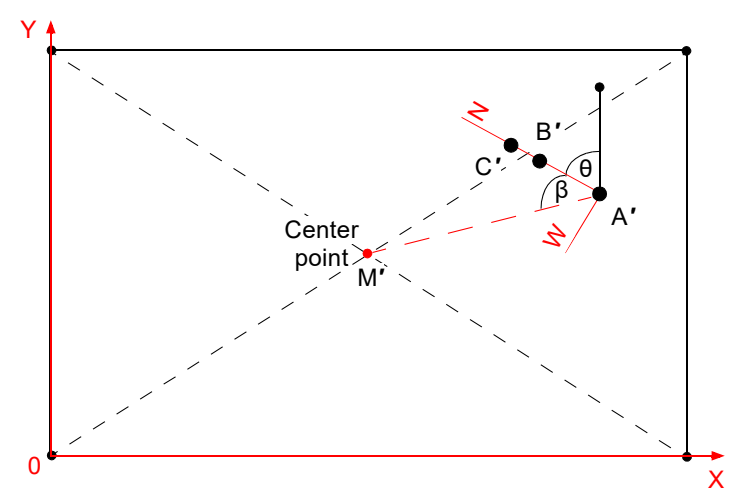

Fig. 4. The camera is located at the ground anywhere.

coordinates of the center point $M$ are $(160,120)$, and the distance $l_{1}$ between $A^{\prime}$ and the center point $M$ is as follows:

$$
l_{1}=\sqrt{\left(X_{1}-160\right)^{2}+\left(Y_{1}-120\right)^{2}}
$$

The distance $l_{2}$ between $C^{\prime}$ and $A^{\prime}$ is as follows:

$$
l_{2}=\lambda_{1}+\lambda_{2}
$$

The distance $l_{3}$ between $C^{\prime}$ and the center point $M$ is as follows:

$$
l_{3}=\sqrt{\left(X_{3}-160\right)^{2}+\left(Y_{3}-120\right)^{2}}
$$

Parameter $\beta$ can be calculated from the cosine theorem

$$
\begin{aligned}
& \cos \beta=\frac{l_{2}^{2}+l_{3}^{2}-l_{1}^{2}}{2 l_{2} l_{3}} \\
& \beta=\operatorname{acos} \frac{l_{2}^{2}+l_{3}^{2}-l_{1}^{2}}{2 l_{2} l_{3}}
\end{aligned}
$$

Assuming that the actual motion step size of the lens is proportional to $l_{3}$, the actual lens movement distance and the pixel point correspondence are as follows:

$$
L=n l_{3}
$$

where $L$ is the distance that the actual lens moves in space, $l_{3}$ is the distance between $C^{\prime}$ and the center point $M$, and $n$ is a constant.

3) Finally calculate the current coordinates of the lens. As shown in Fig. 4, the lens orientation is north $(\mathrm{N})$ east $(\mathrm{E}) \theta$ degrees, north $(\mathrm{N})$ west $(\mathrm{W})$ of the origin $\beta$ degrees and the distance from the origin is $L$. At this point, the lens positioning is shown in Fig. 5. 


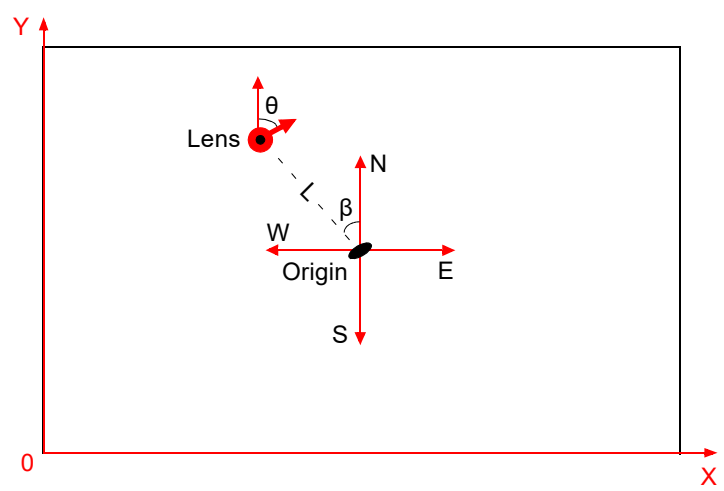

Fig. 5. Actual lens in the floor plan.

Therefore, the current coordinates of the lens can be calculated as follows:

$$
\begin{aligned}
& X_{\text {camera }}=L \sin (-\beta) \\
& Y_{\text {camera }}=L \cos (-\beta)
\end{aligned}
$$

\section{Experiment and discussion}

In order to verify the practicability and reliability of the proposed algorithm, the indoor positioning experiment system based on visible light is designed as Fig. 6 . The visible light indoor positioning experiment system is composed of the light source generating portion and the position detecting portion [13]. The light source generating circuit mainly realizes the illumination of white LED and the control of light intensity, and the light source selects the $5 \mathrm{~mm}$ straw hat white LED which light intensity is large, and which light is concentrated. Even in the environment of heavy smoke, its light penetration is strong, the illumination viewing angle is obvious, the reliability is high, and

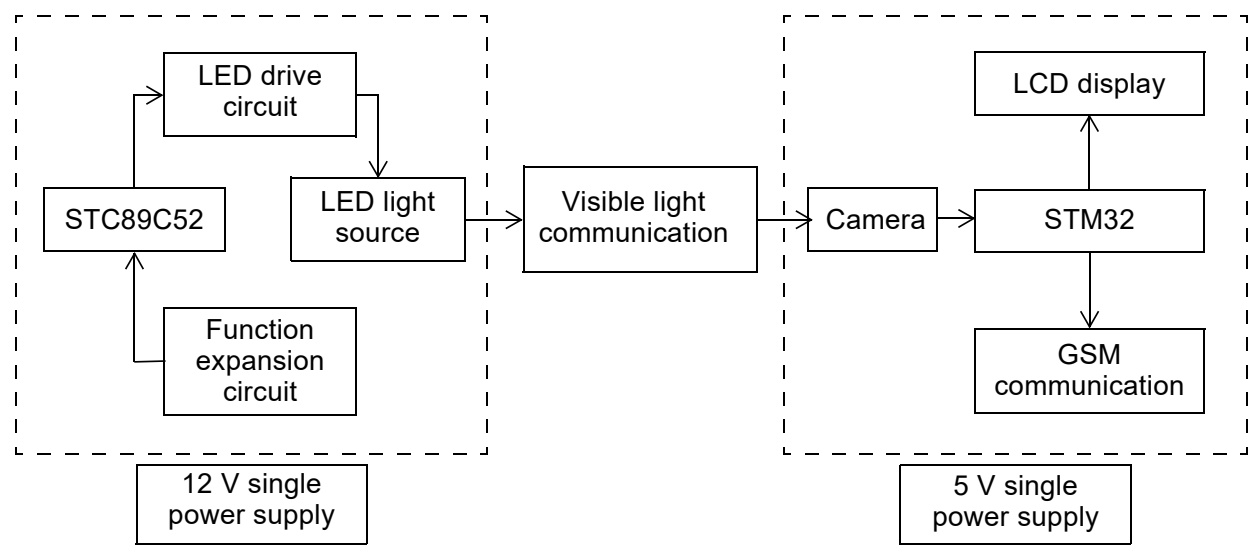

Fig. 6. System composition block diagram. 
the imaging is easy to detect. The position detection adopt scheme is that the digital camera combines with FIFO (first input first output). In addition, the control circuit is simple, the price is relatively cheap, and the digital signal is convenient for circuit design and control relative to the analog signal. Furthermore, the position detection circuit not only needs the position detection process, but also needs the function of wireless data transmission that is realized by GSM [14] short message mode.

\subsection{Hardware design of experiment system}

The whole system is composed of the power module, the image position detecting module, the GSM communication module, the LED light source control module, the display module and so on. This part focuses on the image position detection module.

T a b 1 e 1. Signal of OV7670 digital camera and its description.

\begin{tabular}{ll}
\hline Signal & Function description \\
\hline VCC3.3 & 3.3 V power supply \\
GND & Ground pin \\
OV_SCL & SCCB communication clock signal \\
OV_SDA & SCCB communication data signal \\
FIFO_D[7:0] & FIFO output data \\
FIFO_RCLK & Read FIFO clock \\
FIFO_WEN & FIFO write enable \\
FIFO_WRST & FIFO write pointer reset \\
FIFO_RRST & FIFO read pointer reset \\
FIFO_OE & FIFO output enable (chip select) \\
OV_VSYNC & OV7670 frame sync signal \\
\hline
\end{tabular}

The key to the image position detection module is the design of the digital camera circuit. In this paper, the OV7670 digital camera is selected [15], the LED light source is imaged by the camera, and then the image data is sent to the CPU. The CPU performs binarization processing [16] and then performs position calculation, and finally obtains coordinate information. The external communication signals are shown in Table 1.

\subsection{Software design of experiment system}

The light source part of the system mainly realizes the illumination control of the LED lamp. The main program flow chart of the system is shown in Fig. 7.

In image processing, this paper defines the pixels to be processed. Each two bytes constitutes the color of one pixel (high byte first, low byte last), using gray value calculation formula [17] as follows:

$$
\begin{aligned}
\text { Gray }= & ((((\text { color }) \& 0 \times \mathrm{xF} 800) \gg 8) \times 77+(((\text { color }) \& 0 \mathrm{x} 07 \mathrm{E} 0) \gg 3) \times 105 \\
& +(((\text { color }) \& 0 \mathrm{x} 001 \mathrm{~F}) \ll 3) \times 29+128) / 256
\end{aligned}
$$




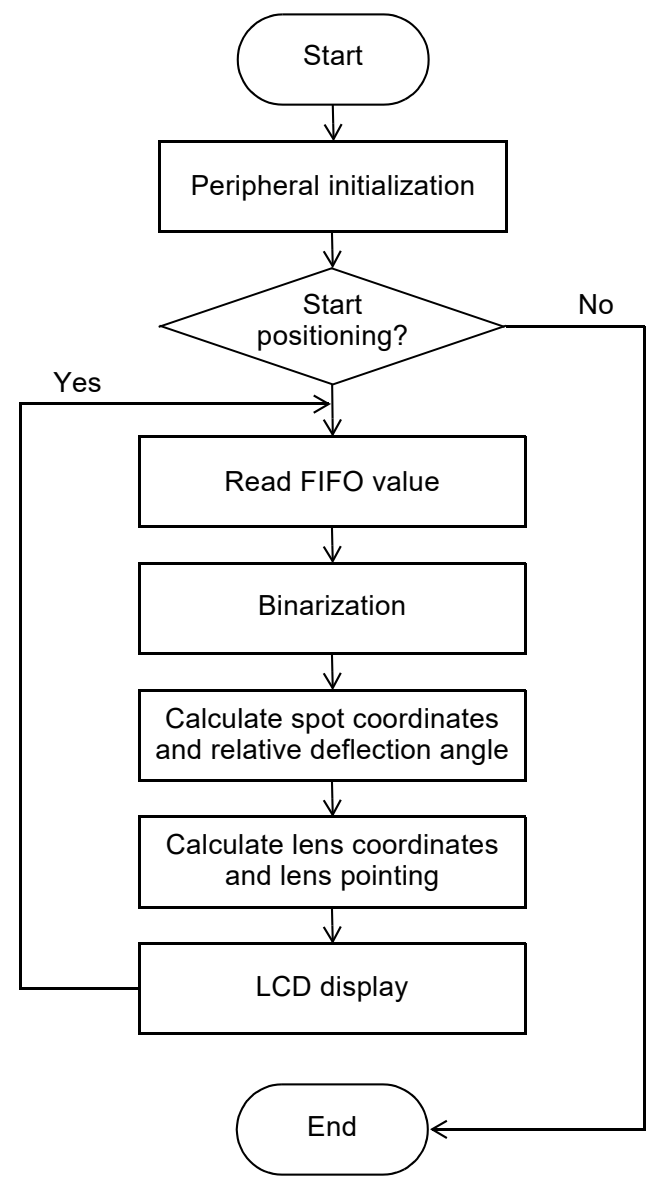

Fig. 7. System program flow chart.

According to the above formula (14), the gray value of the point can be calculated. The point where the gradation value is greater than 150 is a black point, and the point where the gradation value is less than 150 is a white point, and image binarization can be realized.

\subsection{Test and discussion}

The indoor positioning experiment system based on visible light was designed as Fig. 8 . Figure $8 \mathbf{a}$ show the coordinate measurement in the natural environment, and Fig. $8 \mathbf{b}$ show the coordinate measurement in the smoke environment.

The experiment was conducted by making a prototype. In a confined space, the light source part of the system is fixed in the ceiling of the indoor space and its position points to the geographical north direction and is arranged in a straight line. The position detecting part is placed at the coordinates of the bottom of the space, and the test result 


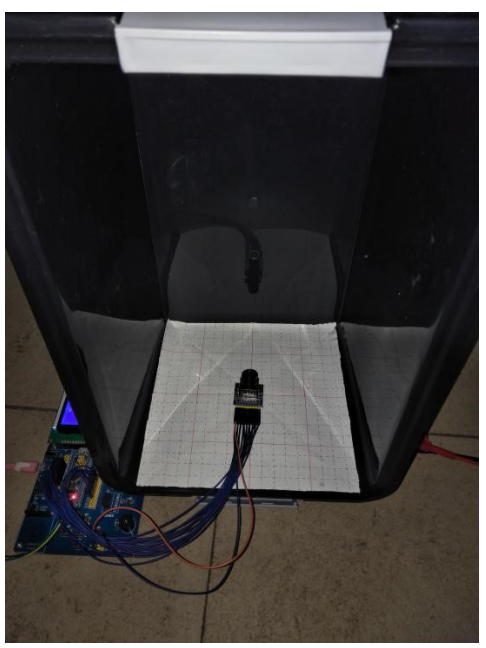

a

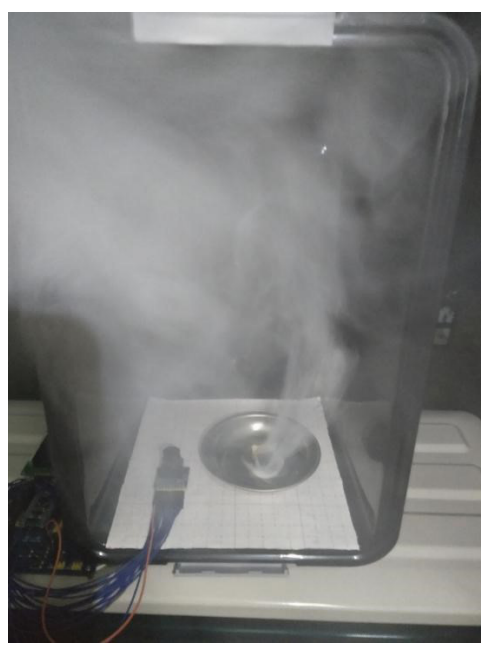

b

Fig. 8. Prototype simulation environment experiment: natural (a) and smoke (b).

is displayed on the LCD, which is compared with the actual result of the coordinate reading.

\subsubsection{Test method}

Place the position detection section on the bottom plane of the confined space and install the three LEDs as described above. Create a Cartesian coordinate system with the center of the plane area where the lens is located as the origin, and divide the plane into five areas: A, B, C, D, and E, as shown in Fig. 9.

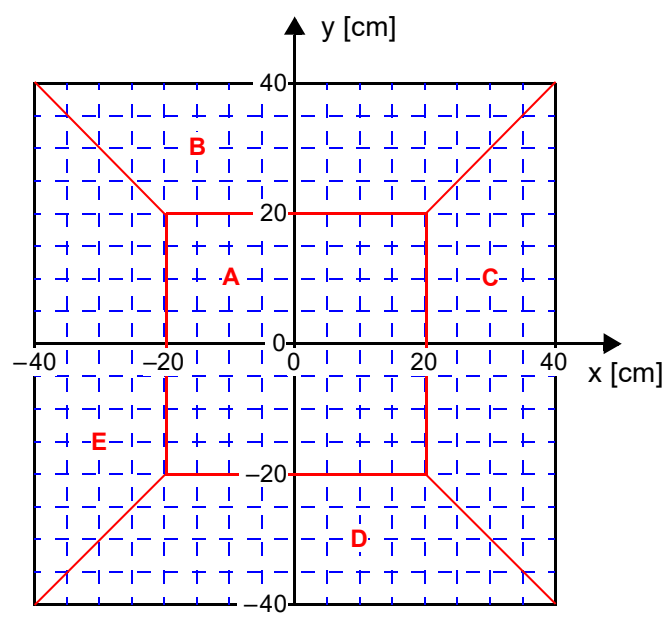

Fig. 9. The area map of the lens. 


\subsubsection{Test results in natural environment}

As shown in Fig. 8a, when the sensor is located in the B and D regions, the measuring circuit can correctly distinguish the upper and lower regions of the abscissa axis and can measure the specific coordinate information. When the sensor is located in the $\mathrm{C}$ and $\mathrm{E}$ areas, the measuring circuit can accurately distinguish the left and right areas of the ordinate axis, and can also measure the specific coordinate information. When it is placed in the A area, the measurement results are shown in Table 2.

$\mathrm{T}$ a b $1 \mathrm{e}$ 2. Coordinates position in natural environment.

\begin{tabular}{llll}
\hline No. & Actual coordinates $[\mathrm{cm}]$ & Measuring coordinates $[\mathrm{cm}]$ & Error $[\mathrm{cm}]$ \\
\hline 1 & $(0,0)$ & $(0.0,0.2)$ & 0.44 \\
2 & $(5,5)$ & $(4.8,4.6)$ & 0.44 \\
3 & $(-20,15)$ & $(-18.8,16.4)$ & 1.84 \\
4 & $(-30,-25)$ & $(-29.4,-24.8)$ & 0.63 \\
5 & $(35,-20)$ & $(32.2,-20.4)$ & 2.83 \\
6 & $(-10,30)$ & $(-8.2,28.4)$ & 2.41 \\
7 & $(23,16)$ & $(21.4,17.2)$ & 2.00 \\
\hline
\end{tabular}

According to the test data, it can be seen that the position measurement can be realized. Among the selected seven sets of coordinates, the largest measurement error reached $2.83 \mathrm{~cm}$ and the smallest measurement error was only $0.44 \mathrm{~cm}$. In short, the measurement error was no more than $3 \mathrm{~cm}$, which indicates that the closer the sensor is placed to the origin, the smaller the positioning error is; the farther away from the origin, the larger the positioning error is. In addition, errors on the boundaries of the five regions $\mathrm{ABCDE}$ need to be considered. When the camera is on the boundary of the A area, there may be a large error due to being far away from the origin, or the error may be smaller because the position of the LED lamp is better. When the camera is on the boundary of the four areas of the BCDE (on the diagonal of the area), the distance from the origin suddenly becomes larger and increases the error, or more likely because the camera can only capture images of two or one of the LED lights. But due to the lens focal length of the camera, there is a certain error in the measurement.

\subsubsection{Test results in smoke environment}

As shown in Fig. 8b, the experiment verifies the feasibility of the proposed algorithm in smoke environment by measuring 6 sets of coordinate precision, and the corresponding smoke concentrations are 1000,5000 and $10000 \mathrm{ppm}$, respectively.

According to the test results of Table 3, it can be known that when the smoke concentration increases, the overall positioning error also increases, but at the highest smoke concentration set, the maximum error is $0.70 \mathrm{~cm}$. When the smoke concentration of the smoke is $1000 \mathrm{ppm}$, the positioning error does not exceed $0.4 \mathrm{~cm}$, and the 
$\mathrm{T}$ a b 1 e 3. Coordinates position in smoke environment.

\begin{tabular}{llllllll}
\hline No. & $\begin{array}{l}\text { Actual } \\
\text { coordinates } \\
{[\mathrm{cm}]}\end{array}$ & $\begin{array}{l}\text { Smoke } \\
\text { concentration } \\
(1000 \mathrm{ppm})\end{array}$ & $\begin{array}{l}\text { Error } \\
{[\mathrm{cm}]}\end{array}$ & $\begin{array}{l}\text { Smoke } \\
\text { concentration } \\
(5000 \mathrm{ppm})\end{array}$ & $\begin{array}{l}\text { Error } \\
{[\mathrm{cm}]}\end{array}$ & $\begin{array}{l}\text { Smoke } \\
\text { concentration } \\
(10000 \mathrm{ppm})\end{array}$ & $\begin{array}{l}\text { Error } \\
{[\mathrm{cm}]}\end{array}$ \\
\hline 1 & $(0,0)$ & $(0.2,0.2)$ & 0.28 & $(0.2,0.3)$ & 0.36 & $(0.3,0.4)$ & 0.50 \\
2 & $(5,5)$ & $(5.2,4.9)$ & 0.22 & $(5.2,4.9)$ & 0.22 & $(5.3,5.0)$ & 0.30 \\
3 & $(8,6)$ & $(7.8,6.1)$ & 0.22 & $(7.9,6.1)$ & 0.14 & $(7.5,6.3)$ & 0.58 \\
4 & $(-7,-5)$ & $(-6.8,-4.8)$ & 0.28 & $(-6.8,-4.8)$ & 0.28 & $(-6.8,-5.0)$ & 0.20 \\
5 & $(8,-7)$ & $(7.9,-6.8)$ & 0.22 & $(7.8,-6.9)$ & 0.22 & $(7.9,-6.7)$ & 0.31 \\
6 & $(-5,8)$ & $(-4.8,7.7)$ & 0.36 & $(-4.8,7.6)$ & 0.44 & $(-4.5,7.5)$ & 0.70 \\
\hline
\end{tabular}

positioning accuracy is high. When the concentration of smoke is $5000 \mathrm{ppm}$, the positioning error is increased compared with the smoke concentration of $1000 \mathrm{ppm}$, but the positioning error is controlled within $0.5 \mathrm{~cm}$. When the concentration of smoke is $10000 \mathrm{ppm}$, the positioning error is up to $0.7 \mathrm{~cm}$, which is much higher than the positioning error of the other two smoke concentrations, but within an acceptable range. In addition, considering the uneven distribution of smoke caused by the artificial smoke environment, it may result in a lower positioning error than the low smoke concentration at high smoke concentrations, which is also within the allowable range.

\subsubsection{Discussion}

In natural environment and in smoke environment, the position coordinates are respectively measured, according to the designed prototype. From the results in Table 2, it can be seen that the positioning error is in the centimeter level which can be used for indoor positioning in natural environment. In addition, different smoke concentration environments are considered to simulate fire rescue scenarios. It can be known from Table 3 that the increase in smoke concentration will slightly increase the positioning error, but the error is within the controllable range which can be applied to fire safety rescue.

Based on the above algorithms and test results, consider preparing a helmet that can be worn by the trapped person when the environment is on fire. A camera is installed on top of the helmet to obtain the image of the LED lights, which can realize the indoor positioning of the trapped person in the skyscraper. This can facilitate the rescue work and improves the life safety factor of the rescue personnel and therefore has high practical application value.

\section{Conclusion}

From the perspective of ensuring life safety, combined with the high-speed time response and modulation characteristics of white LEDs, the visible light indoor positioning algorithm based on fire safety is proposed. In this paper, the geometric relationship between LED lights and image sensors is used to establish a mathematical model to solve 
the positioning problem of the terminal. In addition, this paper also verifies the practicability and reliability of the algorithm by designing the visible light indoor positioning system based on fire safety rescue in natural environment and smoke environment. The system adopts the digital image sensing mode, has high positioning accuracy, fast time response, and can provide people's face orientation information, and it can also carry out wireless short message communication, which can be applied to real-life security rescue. However, fast moving objects [18] are still hard to locate and the system data transmission rate [19] needs to be improved due to the large amount of image data.

Acknowledgments - The authors acknowledge the Hunan Province Science and Technology Program Project (Grant: 2016WK2023).

Data availability statement - The data in the article can be used by anyone, but the related code is subjected to non disclosure agreements and the core product technology needs to be protected.

\section{References}

[1] LuO J., FAN L., Li H., Indoor positioning systems based on visible light communication: state of the art, IEEE Communications Surveys and Tutorials 19(4), 2017, pp. 2871-2893, DOI: 10.1109/ COMST.2017.2743228.

[2] Guan W., Wu Y., Wen S., Chen Y., Chen H., Indoor positioning technology of visible light communication based on CDMA modulation, Acta Optica Sinica 36(11), 2016, article 1106006.

[3] Xu H., Ding Y., Li P., Wang R., Li Y., An RFID indoor positioning algorithm based on Bayesian probability and K-nearest neighbor, Sensors 17(8), 2017, article 1806, DOI: 10.3390/s17081806.

[4] Xu H., Wu M., Li P., Zhu F., Wang R., An RFID indoor positioning algorithm based on support vector regression, Sensors 18(5), 2018, article 1504, DOI: 10.3390/s18051504.

[5] Jung S.-H., LeE G., HAN D., Methods and tools to construct a global indoor positioning system, IEEE Transactions on Systems, Man, and Cybernetics: Systems 48(6), 2018, pp. 906-919, DOI: $10.1109 /$ TSMC.2016.2626797.

[6] YAng D., Xu B., RAo K., Sheng W., Passive infrared (PIR)-based indoor position tracking for smart homes using accessibility maps and A-star algorithm, Sensors 18(2), 2018, article 332, DOI: 10.3390/ $\underline{\mathrm{s} 18020332 .}$

[7] Noh Y., Yamaguchi H., LeE U., Infrastructure-free collaborative indoor positioning scheme for time-critical team operations, IEEE Transactions on Systems, Man, and Cybernetics: Systems 48(3), 2018, pp. 418-432, DOI: 10.1109/TSMC.2016.2615652.

[8] Gu Z., Guo W., Li C., Zhu X., Guo T., An adaptive method for switching between pedestrian/car indoor positioning algorithms based on multilayer time sequences, Sensors 18(3), 2018, article 711, DOI: $10.3390 / \mathrm{s} 18030711$.

[9] Peng Q., Guan W., Wu Y., Cai Y., XIE C., Wang P., Three-dimensional high-precision indoor positioning strategy using Tabu search based on visible light communication, Optical Engineering 57(1), 2018, article 016101, DOI: 10.1117/1.OE.57.1.016101.

[10] Zhang M., Li F., Guan W., Y. Wu, Xie C., Peng Q., Liu X., A three-dimensional indoor positioning technique based on visible light communication using chaotic particle swarm optimization algorithm, Optik 165, 2018, pp. 54-73, DOI: 10.1016/j.ijleo.2018.03.120.

[11] Keskin M.F., Sezer A.D., GezICI S., Localization via visible light systems, Proceedings of the IEEE 106(6), 2018, pp. 1063-1088, DOI: 10.1109/JPROC.2018.2823500.

[12] Ho S.-W., SAed A.A., Lai L., Sung C.W., Coding and bounds for channel estimation in visible light communications and positioning, IEEE Journal on Selected Areas in Communications 36(1), 2018, pp. 34-44, DOI: 10.1109/JSAC.2017.2774718. 
[13] Chiodo M., Giusto P., Jurecska A., Hsien H.C., Sangiovanni-Vincentelli A., Lavagno L., Hardware-software codesign of embedded systems, IEEE Micro 14(4), 1994, pp. 26-36, DOI: 10.1109 / 40.296155.

[14] Mouly M., Pautet M.-B., The GSM System for Mobile Communications, Telecom Publishing, 1992.

[15] Cao N., Nasir S.B., Sen S., Raychowdhury A., Self-optimizing IoT wireless video sensor node with in-situ data analytics and context-driven energy-aware real-time adaptation, IEEE Transactions on Circuits and Systems I: Regular Papers 64(9), 2017, pp. 2470-2480, DOI: 10.1109/TCSI.2017.2716358.

[16] Sauvola J., Pietikäınen M., Adaptive document image binarization, Pattern Recognition 33(2), 2000, pp. 225-236, DOI: 10.1016/S0031-3203(99)00055-2.

[17] Xu Z.-X., СHAN Y.-H., Improving reversible color-to-grayscale conversion with halftoning, Signal Processing: Image Communication 52, 2017, pp. 111-123, DOI: 10.1016/j.image.2016.12.005.

[18] Zhu A., Qi X., Fan T., Gu Z., Lv Q., Ye D., Huangfu J., Sun Y., Zhu W., Ran L., Indoor localization for passive moving objects based on a redundant SIMO radar sensor, IEEE Journal on Emerging and Selected Topics in Circuits and Systems 8(2), 2018, pp. 271-279, DOI: 10.1109/JETCAS.2018. 2798584.

[19] ZaFAR F., BAKAUl M., PARTHIBAN R., Laser-diode-based visible light communication: toward gigabit class communication, IEEE Communications Magazine 55(2), 2017, pp. 144-151, DOI: 10.1109/ MCOM.2017.1500672CM.

Received March 22, 2019

in revised form May 20, 2019 